\title{
THE PATTERN OF CARDIAC DISEASES AT THE CARDIAC CLINIC OF JIMMA UNIVERSITY SPECIALISED HOSPITAL, SOUTH WEST ETHIOPIA
}

\author{
Belete Habte, $\mathrm{MD}^{1}{ }^{*}$, Fessahaye Alemseged, MD, MPHE ${ }^{2}$, Dawit Tesfaye, $\mathrm{MD}^{1}$
}

ABSTRACT

BACKGROUND: Rheumatic heart disease is the commonest cardiac disease in most sub-Saharan African countries, followed by hypertensive heart disease which is rising along with the other noncommunicable diseases. However the pattern in our setting is not known. This study aimed to determine the pattern of cardiac diseases among adult patients on follow-up at the cardiac follow-up clinic of Jimma University Specialized Hospital.

METHODS: A cross-sectional study was conducted on cardiac patients who are newly enrolled to the cardiac follow up clinic of Jimma university specialized hospital during a five year period from 2003 to 2008. Out of the total 837 cases that were newly enrolled to the clinic in the five year period, 781 patients who had complete record about etiologic diagnosis were included in the study. The data were collected using structured record review checklist. The collected data were then analyzed using SPSS for windows version 12.0.

RESULTS: Rheumatic heart disease was the diagnosis in 256 (32.8\%) of the cardiac cases on follow-up followed by hypertensive heart disease and cardiomyopathy accounting for 189 (24.2\%) and 158 (20.2\%) of cases, respectively. Among Rheumatic heart disease patients; male to female ratio was 0.86:1 and the mean age was 31.4 years. One hundred ninety three (75.4\%) of the cases with rheumatic heart disease had echocardiographic report that showed valve(s) involvements of pure MS in 99 (51.3\%) and combined MS, MR in 49 (25.4\%). Overall, hypertension contributed for a total of 241 (30.9\%) of cardiac patients that included 189 (24.2\%) hypertensive heart disease and 52 (6.7\%) as one major risk factor for ischemic heart disease.

CONCLUSION: Rheumatic, hypertensive and cardiomyopathic heart diseases accounted for more than three-quarters of cardiac diseases in the study population. This study highlighted the need for further study to determine the burden at community setting.

KEYWORDS: Cardiac diseases, Pattern, Jimma

\section{INTRODUCTION}

Epidemiologic transition which is taking place in every part of the world, among all races, ethnic groups, and cultures has resulted in the global rise in cardiovascular disorders (CVD). Today CVD became the most common cause of death accounting for $30 \%$ of deaths worldwide, with $80 \%$ of the burden now occurring in developing countries. Although human immunodeficiency virus/acquired immunodeficiency syndrome (HIV/AIDS) is the leading overall cause of death in the sub Saharan region, CVD is the second leading killer and is the leading among those over the age of 30 year $(1,2)$.

\footnotetext{
${ }^{1}$ Department of Internal Medicine, Jimma University, P.O. Box : 1274, E-mail: beletehabte@gmail.com, *Correspondence author

${ }^{2}$ Department of Biostatistics and Epidemiology, Jimma University
}

Cardiac disease as one of the CVD accounted for significant cases of patient visits although the burden for specific etiologic type varies remarkably between regions of the world, which is largely due to the epidemiologic transition and the different stages in its evolution that a country has to undergo. 
During the course of cardiac disease a subset of them develop heart failure. The vast majority of heart failure causes in sub-Saharan Africa are due to the major non-ischemic causes; with rheumatic heart disease (RHD), hypertensive heart disease (HHD), and cardiomyopathy accounting for over $75 \%$ of cases in most series $(1,3,4,5)$.

RHD is estimated to exist with a prevalence of at least 15.6 million cases, with 282,000 new cases and 233,000 deaths each year (6).The incidence of rheumatic fever had declined in the developed countries. However, RHD are still common and evidence suggested that there is little if any decline in the occurrence of rheumatic fever in the developing world. In the sub-Saharan Africa RHD still is common and does not seem decreasing while HHD and IHD are rising competing with the already existing RHD as cause of heart disease in the area "double burden of pre-transitional and post transitional diseases" (4, 7-9).

Ethiopia as one of the African countries also shares the burden of cardiac disease. Some studies conducted in the country indicated that the two major causes of cardiac disease are RHD and HHD furthermore IHD is on the rise $(10,11,12)$. Determining the current pattern of cardiac disease in our setting is required which can also be considered as the beginning of efforts to put forward in characterization of the most prevailing cardiac diseases in our setting and ultimately improve its detection, treatment and possibly prevention (4). Therefore, this study was carried out to describe pattern of cardiac diseases among patients who started follow-up at Jimma University Specialized Hospital (JUSH) cardiac follow-up clinic.

\section{METHODS AND MATERIALS}

The study setting is Jimma University Specialized Hospital which is located in Jimma town $350 \mathrm{Km}$ Southwest of Addis Ababa. It is the only specialized hospital in Jimma zone serving the majority of peoples living in Jimma City and its surrounding. JUSH gives both in patient and out patient services. As one of the out patient services, the hospital has specialty clinics where patients with specific chronic disease are referred for follow-up. These clinics are staffed with internist, residents and nurses who are trained in specific chronic disease patient follow-up.

A cross-sectional study was employed to describe pattern of cardiac diseases among patients registered and started follow-up at the cardiac referral clinic of JUSH during the period from September 14, 2003 - September 14, 2008. The data collection was conducted from June 10, 2008 till October 9, 2008
The source population for the study was all adult cardiac patients registered at JUSH cardiac follow-up clinic during the period from September 14, 2003 - September 14, 2008. The study population was all the source population whose records were available. Sample size was not determined as all the source population was included in the study.

Data were collected using structured pretested format by a first year Internal Medicine Resident. The format included the card number, the date when the patient get registered to the cardiac follow-up clinic, card number, socio-demographic variables, and patient diagnosis including investigations regarding to the underling cardiac disease etiology. The study participants were selected by their name and card number from the cardiac clinic patient registration book, then individual patient's card was traced and collected to fill the data collection format.

The collected data were cleaned, edited and entered into SPSS for Windows version 12.0. Afterwards it was analyzed using SPSS for windows version 12.0 statistical software program. Descriptive analysis was made to describe the pattern of disease and associations were assessed using Chi-square test. Results were then presented using summary measures and frequency distributions. Associations with p-value $<0.05$ were considered to be significant.

The data quality control measures undertaken include: pre-testing of data collection tools, training of data collectors and supervisors and checking completeness and internal consistencies of data.

Ethical clearance was obtained from the department of internal medicine, Jimma University. Permission was obtained from the hospital officials before data collection.

\section{RESULTS}

Out of the 837 cases with cardiac compliant the records of 56 patients were incomplete for the etiologic diagnosis. As a result a total of 781 newly enrolled cardiac patients' record information was analyzed.

Among the total of 781 newly registered cardiac patients $409(52.4 \%)$ were males and $372(47.6 \%)$ were females. The mean age of patients at the time of enrollment was 43.5 ( $\mathrm{SD} \pm 17.2$ years. Four hundred $(51.9 \%)$ of patients were from rural, 254 $(32.9 \%)$ from urban and $117(15.2 \%)$ from semi urban (Table 1).

Out of 781 cardiac patients 256 (32.8\%) had RHD, 189(24.2\%) HHD 158(20.2\%) cardiomyopathy, 94(12.0\%) IHD, 30(3.8\%) Cor-pulmonale $27(13.5 \%)$ arrhythmia and $27(3.4 \%)$ had other sorts of heart diseases (Table 2). 
Table 1. Socio-demographic characteristic of cardiac patients registered and started follow up at the cardiac clinic of JUSH, during the period from Sep 14, 2003 - Sep 142008.

\begin{tabular}{lcc}
\hline Variables & Frequency & Percent \\
\hline Sex & & \\
Male & 409 & 52.4 \\
Female & 372 & 47.6 \\
\hline Residency* & & \\
Urban & 254 & 32.9 \\
Semi urban & 117 & 15.2 \\
Rural & 400 & 51.9 \\
\hline
\end{tabular}

* Residential area was not documented for 10 patients.

The mean age in years for the respective cardiac disease at the time of registry was $31.4(\mathrm{SD} \pm 13.2)$ for RHD; 51.1 (SD1 \pm 4.4$)$ for HHD, $46.5(\mathrm{SD} \pm 16.0)$ for cardiomyopathy, $57.2(\mathrm{SD} \pm 12.2)$ for IHD, 47.0 $(\mathrm{SD} \pm 16.5)$ for Cor-pulmonale and $33.7(\mathrm{SD} \pm 16.2)$ years for Arrhythmia. There was statistically significant difference in mean age of patients with different types of cardiac disease $(\mathrm{P}<0.001)$. Statistically significant mean age difference between male and females patients was observed among HHD $(\mathrm{p}=0.002)$ and cardiomyopathy $(\mathrm{p}=0.004)$ patients only (Table 3$)$.

Table 2. Cardiac disease distribution among patients registered and started follow up at the cardiac clinic of JUSH, during the period from Sep 14, 2003 - Sep 142008.

\begin{tabular}{lcc}
\hline $\begin{array}{l}\text { Type of heart } \\
\text { disease }\end{array}$ & Frequency & Percent \\
\hline RHD & 256 & 32.8 \\
HHD & 189 & 24.2 \\
Dilated & 158 & 20.2 \\
Cardiomyopathy & & \\
IHD & 94 & 12.0 \\
Cor-pulmonale $^{\S}$ & 30 & 3.8 \\
Arrhythmia & 27 & 3.5 \\
\hline Others & 27 & 3.4 \\
\hline
\end{tabular}

$\S 52 \overline{\text { patients were hypertensive and } 13 \text { were smokers. }}$

${ }^{\psi}$ Pericardial disease, congenital heart disease, degenerative valvular disease

Concerning distribution of sex, males constitute $118(46.1 \%)$ among RHD, 118 (62.4\%) among HHD, 79 (50.0\%) among cardiomyopathy, 57
(60.6\%) among IHD, 13(43.3\%) among Corpulmonale and $14(51.8 \%)$ among arrhythmia patients (Table 4).

Table 4. Cardiac disease distribution by sex and residency among patients registered and started follow up at the cardiac clinic of JUSH, during the period from Sep 14, 2003 - Sep 142008.

\begin{tabular}{lccccc}
\hline Etiology & \multicolumn{2}{c}{ Sex } & \multicolumn{3}{c}{ Residential area } \\
\hline RHD & Male & Female & Urban & Semi-urban & Rural \\
& 118 & 138 & 76 & 39 & 138 \\
HHD & $(46.1 \%)$ & $(53.9 \%)$ & $(30.0 \%)$ & $(15.4 \%)$ & $(54.5 \%)$ \\
& 118 & 71 & 63 & 40 & 84 \\
Cardiomyopathy & $(62.4 \%)$ & $(37.7 \%)$ & $(33.7 \%)$ & $(21.4 \%)$ & $(44.9 \%)$ \\
& 79 & 79 & 43 & 18 & 93 \\
IHD & $(50.0 \%)$ & $(50.0 \%)$ & $(27.9 \%)$ & $(11.7 \%)$ & $(60.4 \%)$ \\
\hline
\end{tabular}


Vol. 20, No. 2 July 2010

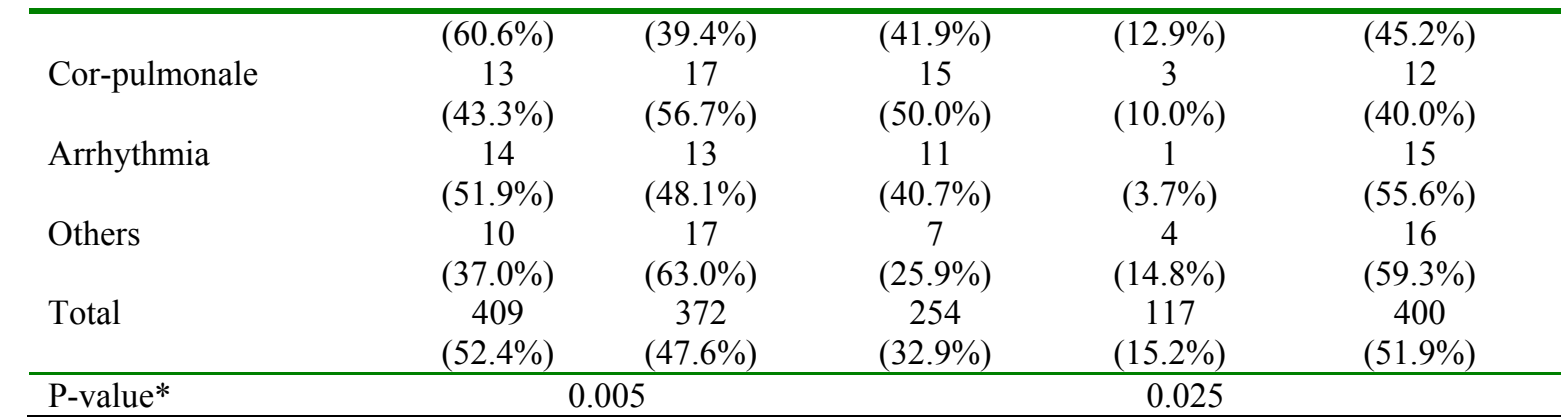

$* \mathrm{p}$-value from Chi-square test

Among 256 RHD patients 193 (75.4\%) patients had Echocardiographic report for the valve(s) involved during the time of enrollment and in the remaining RHD diagnosis were based on clinical and X-ray evidence. The distribution of valvular lesion based on echocardiography included isolated mitral stenosis (MS) in 99 (51.3\%), combined MS and mitral regurgitation (MR) 49(25.4\%), isolated MR and isolated aortic regurgitation (AR) each 12(6.2\%), combined AR and MS 11(5.7\%), and others $10(5.2 \%)$. Concerning sex distribution of patients with major types of valve abnormalities, 44 were males and 55 were females among patients with isolated MS and 22 were males and 27 were females among patients with combined MS and MR. However, the difference in sex distribution was not statistically significant $(\mathrm{P}=0.388)$ (Table $5)$.

\section{DISCUSSION}

This study was carried out to assess the pattern of cardiac disease in the context of ongoing global epidemiologic transition to which Ethiopia is no exception. The pattern of cardiac disease at JUSH where this study is conducted was assessed 5 years back on a sample of 58 patients. The study reported that $72.4 \%$ of cardiac patients were having RHD (13), which should have been an alarm for further studies which need to include screening of high risk individuals so that actual disease prevalence could have been better estimated and subsequent measure could have been done. However, no further studies have been done then after.

The finding of this study would not represent all cardiac cases visiting the hospital as cardiac patients with co-morbid diabetes mellitus were followed at the diabetic clinic and the hospital lacks diagnostic facilities such as cardiac markers. The study was undertaken with such limitations.

The leading four cardiac disease etiologies identified in this study were RHD, HHD, cardiomyopathy, IHD. The finding is similar as compared with the result of other studies done in Ethiopia and Malawi $(10,11,14)$. In the study conducted on the same setting 5 years back, the pattern of cardiac disease in order of decreasing frequency was RHD, Cardiomyopathy, HHD and others (13). The difference could be partly due to difference in case definition and sample size.

Table 3. Mean age distribution by sex of cardiac patients on follow up at the cardiac clinic of JUSH, during the period from Sep 14, 2003 - Sep 14, 2008.

\begin{tabular}{lcccc}
\hline Etiology & \multicolumn{3}{c}{ Sex } & \multirow{2}{*}{ P Value* } \\
\cline { 2 - 4 } & Male & Female & Total & \\
\hline RHD & 1.7 & 31.1 & 31.4 & 0.737 \\
$\quad$ Mean age & 14.1 & 12.4 & 13.2 & \\
$\quad$ SD & & & & \multirow{2}{*}{0.002} \\
$\quad$ MHD & 53.6 & 47.0 & 51.1 & \\
$\quad$ Mean age & 14.7 & 13.1 & 14.4 & \\
Cardiomyopathy & & & & \\
\hline
\end{tabular}




\begin{tabular}{|c|c|c|c|c|}
\hline Mean age & 50.1 & 42.9 & 46.5 & 0.004 \\
\hline SD & 16.7 & 14.4 & 16.0 & \\
\hline \multicolumn{5}{|l|}{ IHD } \\
\hline Mean age & 58.0 & 56.0 & 57.2 & \multirow[t]{2}{*}{0.437} \\
\hline $\mathrm{SD}$ & 11.3 & 13.5 & 12.2 & \\
\hline \multicolumn{5}{|l|}{ Cor-pulmonale } \\
\hline Mean age & 44.2 & 49.1 & 47.0 & \multirow[t]{2}{*}{0.43} \\
\hline $\mathrm{SD}$ & 18.6 & 14.9 & 16.5 & \\
\hline \multicolumn{5}{|l|}{ Arrhythmia } \\
\hline Mean age & 34.8 & 32.5 & 33.7 & \multirow[t]{2}{*}{0.717} \\
\hline $\mathrm{SD}$ & 17.3 & 15.5 & 16.2 & \\
\hline \multicolumn{5}{|l|}{ Others } \\
\hline Mean age & 54.8 & 40.1 & 45.6 & \multirow[t]{2}{*}{0.041} \\
\hline $\mathrm{SD}$ & 14.8 & 18.2 & 18.2 & \\
\hline \multicolumn{5}{|l|}{ Total } \\
\hline Mean age & 46.3 & 40.4 & 43.5 & \multirow[t]{2}{*}{0.000} \\
\hline $\mathrm{SD}$ & 17.9 & 15.9 & 17.2 & \\
\hline P-value $^{\psi}$ & & & 0.000 & \\
\hline
\end{tabular}

Rheumatic heart disease was found to be the leading cause of cardiac disease which is similar with the finding of a study conducted 5 years back on the same setting but the proportion in this study $(32.8 \%)$ is lower than the previous $(72.4 \%)(13)$ which could be due to the actual increase in the other type of heart diseases and partly the difference in the methodology described above may also contribute. It was found that symptomatic RHD patients presented at mean age of 31 years affecting the productive group of the community. Studies showed that lower mean age at development of the diseases was 23 years in Gondar, 25.5 years in Tikur Anbesa Hospital and 24 years in Nigeria $(10,15,16)$. In countries like Ethiopia where surgical intervention for cardiac disease is unavailable adverse outcomes including disability and death of such groups of patients is imminent and the loss to the countries is significant.

Table 5. Pattern of valve(s) involvement among RHD patients registered and started follow up at the cardiac clinic of JUSH, during the period from Sep 14, 2003 - Sep 142008.

\begin{tabular}{lccc}
\hline $\begin{array}{l}\text { Echocardiography report of } \\
\text { valve(s) involved }\end{array}$ & \multicolumn{2}{c}{ Sex } & P-value* \\
\hline & Male & Female & \\
MS & $44(44.4 \%)$ & $55(55.6 \%)$ & \\
MS+MR & $22(44.9 \%)$ & $27(55.1 \%)$ & \\
MR & $6(50.0 \%)$ & $6(50.0 \%)$ & \multirow{2}{*}{0.388} \\
AR & $8(66.7 \%)$ & $4(33.3 \%)$ & \\
AR+MS & $6(54.5 \%)$ & $5(45.5 \%)$ & \\
MR+MS+AR & $3(42.9 \%)$ & $4(57.1 \%)$ & \\
Others & 0 & $3(100 \%)$ & \\
\cline { 1 - 3 } Total & $89(46.1 \%)$ & $104(53.9 \%)$ & \\
\hline
\end{tabular}

*p-value from Chi-square test

Among the RHD patients who had Echo report the Pattern of valve involvement was comparable with other studies $(11,17)$. Overall, MS which is a chronic complication was the identified in $86 \%$ of

patients at the time of first presentation. The presence of MS is evidence for long asymptomatic period which could potentially be intervened by secondary prophylaxis if identified earlier (18). 
Hypertensive heart disease was the second commonest $(24 \%)$ type of heart disease. This is higher as compared to the finding of the previous study done at JUSH $(12 \%)(13)$. In other studies done in developing countries hypertension has become the $1^{\text {st }}$ or the $2^{\text {nd }}$ leading cause of heart disease in the latter case with a progressive narrowing in the magnitude difference between RHD and $\operatorname{HHD}(10,11,12,19)$. Female patients with HHD presented at younger mean age than males. This could be partly due to the fact that males tend to be more active than females ; physical activity have a positive effect with regard to hypertension prevention and it control so at least it delays its progression as found in the South African study (20) which may be the case in our set up too. This issue however needs further study.

Dilated cardiomyopathy accounted for a fifth of cardiac patients and it was the $3^{\text {rd }}$ commonest type of heart disease. Similar findings were noted from similar studies done in developing countries including the previous study done on the same setting $(10,13,14)$. Ischemic heart disease was the fourth leading type of cardiac disease. Majority $(69 \%)$ of them had at least one of the conventional risk factors (hypertension or smoking) of CVD. The proportion of IHD and conventional risk factors is similar with findings of other studies $(14,21,22)$. Therefore identification of cardiovascular disease risk factors and determination of its overall prevalence in our community is required so that both individualized and community based interventions could be implemented $(4,23)$.

In conclusion, this study has found that threequarters of cardiac diseases were due to RHD, HHD and cardiomyopathy in decreasing order of frequency. Statistically significant difference in age at presentation was observed where RHD occurred in the younger productive age group. The proportion of patients with hypertension as cause or contributing factor for cardiac disease has increased. As this study is hospital based with limited representativeness population based studies are recommended

\section{ACKNOWLEDGEMENTS}

We would like to thank Jimma University for funding the study. Our thanks also goes to all those who collaborated in the study.

\section{REFERENCES}

1. Gaziano T. Global burden of cardiovascular disease In: Braunwald's Heart Disease: A Textbook of Cardiovascular Medicine, 8th ed. Philadelphia: Elsevier Saunders, 2007: 1-21.

2. Gaziano T. Cardiovascular disease in the developing world and its cost-effective management. Circulation, 2005; 112(23):35473553.

3. Hunt S, Abraham W, Chin M, et al. ACC/AHA 2005 Guideline Update for the Diagnosis and Management of Chronic Heart Failure in the Adult: a report of the American College of Cardiology/American Heart Association Task Force on Practice Guidelines (Writing Committee to Update the 2001 Guidelines for the Evaluation and Management of Heart Failure): developed in collaboration with the American College of Chest Physicians and the International Society for Heart and Lung Transplantation: endorsed by the Heart Rhythm Society. J Am Coll Cardiol 2005; 112(12): 154-235.

4. Damasceno A, Dzudie A, Mayosi B. Heart failure in sub-Saharan Africa: Time of action. $J$ Am coll cardiol, 2007; 50: 1688-1693.

5. Lopez D. Assessing the burden of mortality from cardiovascular disease. World health stat, 1993; 46: 91-96.

6. Carapetis R, Steer C, Mulholland K, Weber M. The global burden of group A streptococcal diseases. Lancet Infect Dis, 2005; 5(11):68594.

7. Fong W. Infections and cardiovascular system: new perspectives. New York: Kluwer academic publishers, 2004:1-32.

8. Mensah A. Ischemic heart disease in Africa. Heart, 2008; 94(7):836-843.

9. Cooper S, Amoah G, Meensah A. High blood pressure:the foundation for epidemic cardiovascular disease in African population. Ethnicity and disease, 2003; 13(2):48-52.

10. Hodes M. Pattern of heart disease in Ethiopia as seen in a cardiology referral clinic. Cardiology, 1988; 75(6): 456-64.

11. Maru M. The changing pattern of cardiovascular diseases in Ethiopia. East Afr Med J, 1993; 70(12):772-6.

12. Mamo Y, Oli K. Trends of acute myocardial infarction admissions over a decade, Tikur Anbesa Hospital. Ethiop Med J, 2001; 39: 193203.

13. Petros G: Patterns of heart disease Jimma Hospital. Bulletin of JIHS, 1996; 6(2): 85-92.

14. Soliman Z, Juma H. Cardiac disease pattern in Northern Malawi: epidemiologic transition perspective. Epidemiol, 2008; 18(5):204-8.

15. Melka A. Rheumatic heart disease in Gondar college of medical sciences teaching hospital: Socio-demographic and clinical profile. Ethiop Med J, 1996; 34:207_217.

16. Sani U, Karaye M, Borodo M. Prevalence and pattern of rheumatic heart disease in the Nigerian savannah: an echocardiographic study. Cardiovasc J Afr, 2007; 18(5):295-9.

17. Hakim G, Manyemba J. Cardiac disease distribution among patients referred for 
echocardiography in Harare, Zimbabwe. Cent Afr J Med, 1998; 44(6):140-4.

18. Dajani A, Taubert K, Ferrieri P, Peter G, Shulman S. Treatment of acute streptococcal pharyngitis and prevention of rheumatic fever: a statement for health professionals; Committee on Rheumatic Fever, Endocarditis, and Kawasaki Disease of the Council on Cardiovascular Disease in the Young. The American Heart Association Pediatrics, 1995; 96(4):758-64.

19. Amoah G, Kallen C. Etiology of heart failure as seen from a cardiac referral centre in Africa. Cardiology, 2000; 93(1-2):11-18.

20. Kruger S, Venter S, Vorster H. Physical inactivity as a risk factor for cardiovascular disease in communities undergoing rural to urban transition: THUSA study. Cardiovasc $J$ $S$ Afr, 2003; 14(1):16-23.

21. Daviglus L, Lioyd-Jones M, Pirzada A. Preventing cardiovascular disease in the $21^{\text {st }}$ century: Theraputic and preventive implications of current evidence. Am J Cardiovasc Drugs, 2006; 6(2):87-101.

22. Alberts M, Urdal P, Steyn K. Prevalence of cardiovascular diseases and associated risk factors in rural black population of South Africa. Eur J Cardiovasc Prev Rehabil, 2005; 12(4):347-54.

23. Reddy K, Yusuf S. Emerging Epidemic of Cardiovascular Disease in Developing Countries. Circulation, 1998; 97: 596-601. 